\title{
A New Best Approximation Result in (S) Convex Metric Spaces
}

\author{
M. Sabiri, J. Mouline, A. Bassou (D), and T. Sabar \\ Laboratory of Algebra, Analysis and Applications (L3A), Department of Mathematics and Computer Science, \\ Faculty of Sciences Ben M'sik, Hassan II University of Casablanca, P.B. 7955, Sidi Othman, Casablanca, Morocco
}

Correspondence should be addressed to A. Bassou; hbassou@gmail.com

Received 3 December 2019; Revised 27 January 2020; Accepted 12 February 2020; Published 16 March 2020

Academic Editor: Sumit Chandok

Copyright ( 2020 M. Sabiri et al. This is an open access article distributed under the Creative Commons Attribution License, which permits unrestricted use, distribution, and reproduction in any medium, provided the original work is properly cited.

Consider a self-mapping $T$ defined on the union of $p$ subsets of a metric space, and $T$ is said to be $p$ cyclic if $T\left(A_{i}\right) \subseteq A_{i+1}$ for $i=1, \ldots, p$ with $A_{p+1}=A_{1}$. In this article, we introduce the notion of $(S)$ convex structure, and we acquire a best proximity point for $p$ cyclic contraction in $(S)$ convex metric spaces.

\section{Introduction}

Let $\left\{A_{i}\right\}_{i=1}^{p}$ be nonempty subsets of a metric space $(X, d)$. A mapping $T: \cup_{i=1}^{p} A_{i} \longrightarrow \cup_{i=1}^{p} A_{i}$ is said to be cyclic if $T\left(A_{i}\right) \subseteq A_{i+1}$ for $i=1, \ldots, p$ with $A_{p+1}=A_{1}$.

In 2003, Kirk et al. [1] proved that if $T: \cup_{i=1}^{p} A_{i} \longrightarrow$ $\cup_{i=1}^{p} A_{i}$ is cyclic and for some $k \in(0,1), d(T x, T y) \leq$ $k d(x, y)$ for all then $\cap_{i=1}^{p} A_{i} \neq \varnothing$ and $T$ has a unique fixed point in $\cap_{i=1}^{p} A_{i}$. In the case where $\cap_{i=1}^{p} A_{i}=\varnothing$, Eldred and Veeramani [2] introduced the existence of the best proximity point for the map $T$ in setting of uniformly convex Banach spaces.

Theorem 1 (see [2]). Let $A$ and $B$ be nonempty closed and convex subsets of a uniformly convex Banach space. Suppose $T: A \cup B \longrightarrow A \cup B$ is a cyclic contraction map, then there exists a unique best proximity point $x$ in $A$ (that is, with $\|x-T x\|=\operatorname{dist}(A, B))$. Further, if $x_{0} \in A$ and $x_{n+1}=T x_{n}$, then $\left\{x_{2 n}\right\}$ converges to the best proximity point.

This result received considerable interest by many authors recently and more results have been obtained, see for example $[3,4]$.
In 2009, Al-Thagafi and Shahzad [5] studied convergence and existence results of best proximity points for $\varphi$-contraction mappings, and in 2011, Sadiq Basha [6] stated some best proximity point results. We can find other results on best proximity points in [7] by Felhi and Aydi. In [8], results of the best proximity point for cyclic Meir-Keeler contraction mappings were found.

In 2017, T. Sabar et al. [9] studied convergence and existence results of best proximity points for tricyclic contraction.

Theorem 2 (see [10]). Let $A, B$, and $C$ be nonempty closed, bounded, and convex subsets of a $(S)$ convex metric space $(X, d, W)$ which has the $(C)$ property; suppose $A, B$, and $C$ are disjoint subsets of $[a, b]$ where $a, b \in X$, let $T: A \cup B \cup C \longrightarrow A \cup B \cup C$ be a tricyclic contraction map. Then, $T$ has a best proximity point.

In this work, we introduce new results of the best proximity points for a self-mapping defined on the union of $p$ nonempty subsets of a $(S)$ convex metric space $(X, d, W)$.

Let $\left\{A_{i}\right\}_{i=1}^{p}$ be nonempty subsets of a metric space $(X, d)$, then we shall adopt the following notations throughout this paper: 


$$
\begin{aligned}
D_{p}: X^{p} \longrightarrow \mathbb{R}^{+},\left(x_{1}, x_{2}, \ldots, x_{p}\right) \longmapsto D_{p}\left(x_{1}, x_{2}, \ldots, x_{p}\right) & =\sum_{i<j} d\left(x_{i}, x_{j}\right) \quad \text { for } \quad 1 \leq i, \quad j \leq p, \\
\delta\left\{\prod_{i=1}^{p} A_{i}\right\} & =\inf \left\{D_{p}\left(x_{1}, x_{2}, \ldots, x_{p}\right): x_{i} \in A_{i}, \quad i=1, \ldots, p\right\}, \\
\Delta\left\{\prod_{i=1}^{p} A_{i}\right\} & =\sup \left\{D_{p}\left(x_{1}, x_{2}, \ldots, x_{p}\right): x_{i} \in A_{i}, \quad i=1, \ldots, p\right\}, \\
\Delta\left(x_{1}, x_{2}, \ldots, x_{p-1}\right) & \left(A_{p}\right)=\sup \left\{D_{p}\left(x_{1}, x_{2}, \ldots, x_{p}\right): x_{p} \in A_{p}\right\},
\end{aligned}
$$

for all $x_{i} \in A_{i}, i=1, \ldots, p-1$.

Definition 1. Let $\left\{A_{i}\right\}_{i=1}^{p}$ be nonempty subsets of a metric space $(X, d)$, and a mapping $T: \cup_{i=1}^{P} A_{i} \longrightarrow \cup_{i=1}^{P} A_{i}$ is said to be $\mathrm{p}$ cyclic contraction if

(1) $T\left(A_{i}\right) \subseteq A_{i+1}$ for $1 \leq i \leq p$, with $A_{p+1}=A_{1}$

(2) $D_{p}\left(T x_{1}, T x_{2}, \ldots, T x_{p}\right) \leq k D_{p}\left(x_{1}, x_{2}, \ldots, x_{p}\right)+$ $(1-k) \delta\left\{\Pi_{i=1}^{p} A_{i}\right\}$ for some $k \in(0,1)$ and all $x_{i} \in A_{i}, i=1, \ldots, p$

\section{Preliminaries}

Definition 2. Let $\left\{A_{i}\right\}_{i=1}^{p}$ be nonempty subsets of a metric space $(X, d)$.

$T: \cup_{i=1}^{P} A_{i} \longrightarrow \cup_{i=1}^{P} A_{i}$ is a $p$ cyclic contraction; let $x \in \cup_{i=1}^{P} A_{i}$, where $x$ is said to be a best proximity point for $T$ if

$$
D_{p}\left(x, T x, s T^{2} x, \ldots, T^{p-1} x\right)=\delta\left\{\prod_{i=1}^{p} A_{i}\right\} .
$$

Definition 3 (see [11]). Let $(X, d)$ be metric space, then a mapping $W: X \times X \times I \longrightarrow X$ is to be a convex structure on $X$ provided that

$$
\begin{aligned}
& d(u, W(x, y, \lambda)) \leq \lambda d(u, x)+(1-\lambda) d(u, y), \\
& \text { for all } u, x, y \in X \text { and } \lambda \in I:=[0,1] .
\end{aligned}
$$

A metric space $(X, d)$ along with a convex structure $W$ is called a convex metric space and is denoted by $(X, d, W)$, and we denote by $[x, y]$ the set $\{W(x, y, \lambda): \lambda \in I\}$. A subset $C$ of a convex metric space is said to be a convex if $[x, y] \subseteq C$ for all $x, y \in C$. The closed and convex hull of set $A$ will be denoted by

$\overline{c o}(A):=\cap\{C: C$ is a closed and convex subset of $X$ that contains $A$ \}.

Definition 4 (see [12]). A normed linear space $X$ is said to have the property $(C)$ if every bounded decreasing net of nonempty closed and convex subsets of $X$ has a nonempty intersection.

For example, a reflexive Banach space has the property (C), so does every weakly compact convex subset of Banach.
Definition 5. Let $x_{1}, x_{2}, \ldots, x_{p-1}$ be points of a convex metric space $(X, d, W)$ and $r>0$ the closed ball (resp. opened) of focuses $x_{1}, x_{2}, \ldots, x_{p-1}$, then ray $r$ is defined by

$$
\begin{aligned}
& B\left(x_{1}, x_{2}, \ldots, x_{p-1}, r\right) \\
& \quad=\left\{x \in X: D_{p}\left(x_{1}, x_{2}, \ldots, x_{p-1}, x 0\right) \leq(\text { resp }<) r\right\} .
\end{aligned}
$$

Remark 1. $B\left(x_{1}, x_{2}, \ldots, x_{p-1}, r\right)$ is bounded, closed, and convex.

Definition 6 (see [4]). Let $(X, d, W)$ be a convex metric space, in which $\mathrm{W}$ is said to be a strict convex structure if it has the property that whenever $w \in X$ there is $(x, y, \lambda) \in X \times X \times I$ for which

$$
d(u, w) \leq \lambda d(u, x)+(1-\lambda) d(u, y)
$$

for all $u \in X$ then $w=W(x, y, \lambda)$. If $\mathrm{W}$ is a strict convex structure on $(X, d)$, the $(X, d, W)$ is called a strictly convex metric space.

Lemma 1 (see [4]). Let $(X, d, W)$ be a strictly convex metric space; then, for every $\left(x, y, \lambda_{1}, \lambda_{2}\right) \in X^{2} \times I^{2}$ we have $W\left(W\left(x, y, \lambda_{1}\right), y, \lambda_{2}\right)=W\left(x, y, \lambda_{1} \lambda_{2}\right)$.

Definition 7 (see [4]). Let $(X, d, W)$ be a convex metric space. $\mathrm{W}$ is to be called a $(S)$ convex structure on $X$ provided that whenever $x, y \in X$ such that $x=W(a, b, \alpha)$ and $y=$ $W(a, b, \beta)$ where $\alpha>\beta$ and $a, b \in X$, we have $x=W(a, y,((\alpha-\beta) /(1-\beta)))$ and $y=W(x, b,(\beta / \alpha))$.

If $W$ is a $(S)$ convex structure on $X$, then $(X, d, W)$ is called a $(S)$ convex metric space.

Proposition 1. Let $(X, d, W)$ be a $(S)$ convex metric space and let $x, y \in X$ such that $x=W(a, b, \alpha), y=$ $W(a, b, \beta)$, and $z=W(a, b, \gamma)$ where $a, b \in X$ and $\alpha>\beta>\gamma$, then $d(x, y)=(\alpha-\beta) d(a, b)$ and $y=W(x, z,((\beta-\gamma) /$ $(\alpha-\gamma)))$.

Proof. By the $(S)$ property, we have $x=W(a, y,((\alpha-\beta) /$ $(1-\beta)))$, 


$$
\begin{aligned}
d(x, y) & =d\left(W\left(a, y,\left(\frac{(\alpha-\beta)}{(1-\beta)}\right)\right), y\right) \\
& =\left(\frac{(\alpha-\beta)}{(1-\beta)}\right) d(a, y)=\left(\frac{(\alpha-\beta)}{(1-\beta)}\right) d(a, W(a, b, \beta)) \\
& =(\alpha-\beta) d(a, b) .
\end{aligned}
$$

Since $\alpha>\gamma$ and $\beta>\gamma$, then $x=W(a, z,((\alpha-\gamma) /$ $(1-\gamma)))$ and $y=W(a, z,((\beta-\gamma) /(1-\gamma)))$ and put $\alpha^{\prime}=$ $((\alpha-\gamma) /(1-\gamma))$ and $\beta \prime=((\beta-\gamma) /(1-\gamma))$, then we have $y=W(x, z,(\beta \prime / \alpha \prime))=W(x, z,((\beta-\gamma) /(\alpha-\gamma)))$.

Now, let $\left(X_{i}, d_{X_{i}}, W_{X_{i}}\right), i=1, \ldots, p-1$ be convex metric spaces, then the mapping

$$
d_{1}: \prod_{i=1}^{p-1} X_{i} \times \prod_{i=1}^{p-1} X_{i} \longrightarrow \mathbb{R}^{+}
$$

defined by

$$
d_{1}\left(\left(x_{1}, x_{2}, \ldots, x_{p-1}\right),\left(y_{1}, y_{2}, \ldots, y_{p-1}\right)\right)=\sum_{i=1}^{p-1} d_{X_{i}}\left(x_{i}, y_{i}\right)
$$

is a distance on $\Pi_{i=1}^{p-1} X_{i}$.

\section{Main Results}

Before presenting our results, we give the following lemma.

Lemma 2. The mapping

$$
W\left(\prod_{i=1}^{p-1} X_{i}\right):\left(\prod_{i=1}^{p-1} X_{i}\right) \times\left(\prod_{i=1}^{p-1} X_{i}\right) \times I \longrightarrow\left(\prod_{i=1}^{p-1} X_{i}\right)
$$

defined by

$$
\begin{aligned}
& \left.W\left(\prod_{i=1}^{p-1} X_{i}\right)\left(\left(x_{1}, x_{2}, \ldots, x_{p-1}\right),\left(y_{1}, y_{2}, \ldots, y_{p-1}\right)\right), \lambda\right) \\
& \quad=\left(W_{X_{1}}\left(x_{1}, y_{1}, \lambda\right), W_{X_{2}}\left(x_{2}, y_{2}, \lambda\right), \ldots, W_{X_{p-1}}\left(x_{p-1}, y_{p-1}, \lambda\right)\right),
\end{aligned}
$$

is a convex structure on the metric space $\left(\Pi_{i=1}^{p-1} X_{i}, d_{1}\right)$.

Proof. Let

$$
\begin{aligned}
\left(u_{1}, u_{2}, \ldots, u_{p-1}\right) \in\left(\Pi_{i=1}^{p-1} X_{i}\right),\left(x_{1}, x_{2}, \ldots, x_{p-1}\right),\left(y_{1}, y_{2}, \ldots, y_{p-1}\right) \in\left(\Pi_{i=1}^{p-1} X_{i}\right), \quad \lambda \in I \\
d_{1}\left(\left(u_{1}, u_{2}, \ldots, u_{p-1}\right), W\left(\begin{array}{c}
p-1 \\
\pi X_{i} \\
i=1
\end{array}\right)\left(\left(x_{1}, x_{2}, \ldots, x_{p-1},\left(y_{1}, y_{2}, \ldots, y_{p-1}\right), \lambda\right)\right)\right) \\
=d_{1}\left(\left(u_{1}, u_{2}, \ldots, u_{p-1}\right),\left(W_{X_{1}}\left(x_{1}, y_{1}, \lambda\right), W_{X_{2}}\left(x_{2}, y_{2}, \lambda\right), \ldots, W_{X_{p-1}}\left(x_{p-1}, y_{p-1}, \lambda\right)\right)\right. \\
=\sum_{i=1}^{p-1} d_{X_{i}}\left(u_{i}, W_{X i}\left(x_{i}, y_{i}, \lambda\right)\right) \leq \sum_{i=1}^{p-1} \lambda d_{X_{i}}\left(u_{i}, x_{i}\right)+\sum_{i=1}^{p-1}(1-\lambda) d_{X_{i}}\left(u_{i}, y_{i}\right) \\
\leq \lambda \sum_{i=1}^{p-1} d_{X_{i}}\left(u_{i}, x_{i}\right)+(1-\lambda) \sum_{i=1}^{p-1} d_{X_{i}}\left(u_{i}, y_{i}\right) \\
=\lambda d_{1}\left(u_{1}, u_{2}, \ldots, u_{p-1},\left(x_{1}, x_{2}, \ldots, x_{p-1}\right)\right. \\
\quad+(1-\lambda) d_{1}\left(u_{1}, u_{2}, \ldots, u_{p-1},\left(y_{1}, y_{2}, \ldots, y_{p-1}\right) .\right.
\end{aligned}
$$

Definition 8. A subset $E$ of the convex metric space $\left(\Pi_{i=1}^{p-1} X_{i}, d_{1}, W\left(\Pi_{i=1}^{p-1} X_{i}\right)\right)$ is a convex if

$$
W\left(\Pi_{i=1}^{p-1} X_{i}\right)\left(\left(x_{1}, x_{2}, \ldots, x_{p-1}\right),\left(y_{1}, y_{2}, \ldots, y_{p-1}\right), \lambda\right) \in E,
$$

for all $\left(x_{1}, x_{2}, \ldots, x_{p-1}\right),\left(y_{1}, y_{2}, \ldots, y_{p-1}\right) \in E$ and $\lambda \in I$. We now state our first main result.

Theorem 3. Let $\left\{A_{i}\right\}_{i=1}^{p}$ be nonempty, closed, bounded, and convex subsets of a $(S)$ convex metric space $(X, d, W)$ which has the $(C)$ property; suppose $\left\{A_{i}\right\}_{i=1}^{p}$ are disjoint subsets of $[a, b]$ where $a, b \in X$, let $a$ map $T: \cup_{i=1}^{p} A_{i} \longrightarrow \cup_{i=1}^{p} A_{i}$ be $p$ cyclic contraction map. Then, $T$ has a best proximity point.

Proof. We denote by $\sum$ the set of all nonempty, bounded, closed, and convex subset $\left(B_{1}, B_{2}, \ldots, B_{p}\right) \subseteq\left(A_{1}, A_{2}, \ldots, A_{p}\right)$ such that $T$ is p cyclic on $\cup_{i=1}^{P} B_{i}$. Then, $\sum$ is nonempty and partially ordered by reverse inclusion, that is,

$$
B_{1 i} \leq B_{2 i} \Longleftrightarrow B_{2 i} \subseteq B_{1 i} . \quad \text { For } i=1,2, \ldots, p .
$$


Let $\left(B_{i j}\right)_{j \in I i=1, \ldots, p}$ be an increasing chain of $\sum$, and since $X$ has the property $(C), \cap_{j \in I} B_{i j}$ for ${ }_{i=1, \ldots, p}$ are bounded, closed, and convex. We have

$$
B_{i j} \leq \bigcap_{j \in I} B_{i j} \text { for }_{i=1, \ldots, p} \text { for all } j \in I .
$$

By Zorn's lemma, we have a maximal element say $\left(C_{1}, C_{2}, \ldots \ldots C_{p}\right) \in \sum$. We have

$$
\overline{c o}\left(T\left(C_{i}\right)\right) \subseteq C_{i+1}, \quad \text { for } i=1,2, \ldots, p \text { with } C_{p+1}=C_{1} \text {, }
$$

hence, $T\left(\overline{c o}\left(T\left(C_{i}\right)\right)\right) \subseteq T\left(C_{i+1}\right) \subseteq \overline{c o}\left(T\left(C_{i+1}\right)\right)$; we have $T$ which is $p$ cyclic on $\cup_{i=1}^{p}\left(\overline{c o}\left(T\left(C_{i}\right)\right)\right.$, and by the maximality of $\left(C_{1}, C_{2}, \ldots, C_{p}\right)$ we have

$$
C_{i+1}=\overline{c o}\left(T\left(C_{i}\right)\right), \quad \text { for } i=1,2, \ldots, p .
$$

Now let $x_{i} \in C_{i}$ for $i=1,2, \ldots, p, k \in(0,1)$ :

$$
\begin{aligned}
D_{p}\left(T x_{1}, \ldots, T x_{p}\right) & \leq k D_{p}\left(x_{1}, \ldots, x_{p}\right)+(1-k) \delta\left(\prod_{i=1}^{p} A_{i}\right) \\
& \leq k \Delta\left(C_{1}, \ldots, C_{p}\right)+(1-k) \delta\left(\prod_{i=1}^{p} A_{i}\right)=\Lambda .
\end{aligned}
$$

Then, $T x_{p} \in B\left(T x_{1}, \ldots, T x_{p-1}, \Lambda\right) \forall x_{p} \in C_{p} \Rightarrow T\left(C_{p}\right) \subset$ $B\left(T x_{1}, \ldots, T x_{p-1}, \Lambda\right) . C_{1}=\overline{c o}\left(T\left(C_{p}\right)\right) \subset B\left(T x_{1}, \ldots, T x_{p-1}\right.$, $\Lambda)$ so $\Delta_{\left(T x_{1}, \ldots, T x_{p-1}\right)}\left(C_{1}\right) \leq \Lambda$.

Put

$$
\begin{aligned}
& B_{1}=\left\{\left(x_{2}, \ldots, x_{p}\right) \in\left(\prod_{i=2}^{p} C_{i}\right): \Delta x_{\left(x_{2}, \ldots, x_{p}\right)}\left(C_{1}\right) \leq \Lambda\right\}, \\
& B_{i}=\left\{\left(x_{i+1}, \ldots . . x_{p+i-1}\right) \in\left(\prod_{j=i+1}^{p+i-1} C_{j}\right): \Delta \Delta_{\left(x_{i+1}, \ldots . . x_{p+i-1}\right)}\left(C_{i}\right) \leq \Lambda\right\},
\end{aligned}
$$

for $i=2, \ldots, p$ with $C_{p+j}=C_{j}$ for $j=1, \ldots, p . B_{i}$ is nonempty, bounded, and closed for $i=1,2, \ldots, p$.

Put $\Psi_{x_{1}}: \prod_{i=2}^{p} C_{i} \longrightarrow \mathbb{R}^{+}$and let $x_{1} \in C_{1}$ such that $\Psi_{x_{1}}\left(x_{2}, \ldots, x_{p}\right)=D_{p}\left(x_{1}, \ldots, x_{p}\right)$, then we have

$$
B_{1}=\bigcap_{x_{1} \in C_{1}} \Psi_{x_{1}}^{-1}([0, \Lambda]) .
$$

Let $\left(x_{2}, \ldots, x_{p}\right),\left(y_{2}, \ldots, y_{p}\right) \in B_{1}, x_{1} \in C_{1}$ and $\lambda \in I$. We have

$$
\begin{aligned}
D_{p}( & \left.W\left(x_{2}, y_{2}, \lambda\right), W\left(x_{3}, y_{3}, \lambda\right), \ldots, W\left(x_{p}, y_{p}, \lambda\right), x_{1}\right) \\
= & \sum_{2 \leq i<j \leq p} d\left(W\left(x_{i}, y_{i}, \lambda\right), W\left(x_{j}, y_{j}, \lambda\right)\right)+\sum_{i=2}^{p} d\left(W\left(x_{i}, y_{i}, \lambda\right), x_{1}\right) \\
\leq & \sum_{2 \leq i<j \leq p} \lambda d\left(x_{i}, W\left(x_{j}, y_{j}, \lambda\right)+(1-\lambda) \sum_{2 \leq i<j \leq p} d\left(y_{i}, W\left(x_{j}, y_{j}, \lambda\right)+\lambda \sum_{i=2}^{p} d\left(\left(x_{i}, x_{1}\right)+(1-\lambda) \sum_{i=2}^{p} d\left(\left(y_{i}, x_{1}\right)\right.\right.\right.\right. \\
\leq & \lambda \sum_{i=2}^{p} d\left(\left(x_{i}, x_{1}\right)+(1-\lambda) \sum_{i=2}^{p} d\left(\left(y_{i}, x_{1}\right)+\right.\right. \\
& +\lambda \sum_{2 \leq i<j \leq p}\left(\lambda d\left(x_{i}, x_{j}\right)+(1-\lambda) d\left(x_{i}, y_{j}\right)\right)+(1-\lambda) \sum_{2 \leq i<j \leq p}\left(\lambda d\left(y_{i}, x_{j}\right)+(1-\lambda) d\left(y_{i}, y_{j}\right)\right) \\
\leq & \lambda \sum_{i=2}^{p} d\left(x_{i}, x_{1}\right)+(1-\lambda) \sum_{i=2}^{p} d\left(y_{i}, x_{1}\right)+\lambda^{2} \sum_{2 \leq i<j \leq p} d\left(x_{i}, x_{j}\right) \\
& +\lambda(1-\lambda)\left(\sum_{2 \leq i<j \leq p} d\left(x_{i}, y_{j}\right)+d\left(y_{i}, x_{j}\right)\right)+(1-\lambda)^{2} \sum_{2 \leq i<j \leq p} d\left(y_{i}, y_{j}\right) .
\end{aligned}
$$

Since $x_{i}, y_{i} \in[a, b]$ for $i=1, \ldots, p$ implies there exist $\alpha_{i}, \beta_{i} \in I$, such that $d\left(x_{i}, y_{j}\right)=\left|\alpha_{i}-\beta_{j}\right| d(a, b), d\left(x_{i}, x_{j}\right)=$ $\left|\alpha_{i}-\alpha_{j}\right| d(a, b)$, and $d\left(y_{i}, y_{j}\right)=\left|\beta_{i}-\beta_{j}\right| d(a, b)$.

If $\left.\alpha_{i}>\beta_{i}, \alpha_{j} \notin\right] \alpha_{i}, \beta_{i}$ [ and $\left.\beta_{i} \notin\right] \alpha_{j}, \beta_{j}$ [ for $i \neq j$.
Suppose for example $\alpha_{1}<\alpha_{2}<\beta_{1}$, then we have $x_{2}=$ $W\left(y_{1}, x_{1},\left(\left(\alpha_{2}-\alpha_{1}\right) /\left(\beta_{1}-\alpha_{1}\right)\right)\right) \Longrightarrow x_{2} \in\left[y_{1}, x_{1}\right]$ since $C_{1}$ is convex then $\left[y_{1}, x_{1}\right] \subseteq C_{1} \Rightarrow x_{2} \in C_{1}$, which is a contradiction, $C_{1} \cap C_{2}=\varnothing$. 
We have $d\left(x_{i}, y_{j}\right)+d\left(y_{i}, x_{j}\right)=d\left(x_{i}, x_{j}\right)+d\left(y_{i}, y_{j}\right)$.

$$
\begin{aligned}
& \left.D_{p}\left(W\left(x_{2}, y_{2}, \lambda\right), W\left(x_{3}, y_{3}, \lambda\right), \ldots, W\left(x_{p}, y_{p}, \lambda\right), x_{1}\right)\right) \\
& \leq \lambda \sum_{i=2}^{p} d\left(\left(x_{i}, x_{1}\right)+(1-\lambda) \sum_{i=2}^{p} d\left(\left(y_{i}, x_{1}\right)+\lambda^{2} \sum_{2 \leq i<j \leq p} d\left(x_{i}, x_{j}\right)\right.\right. \\
& \quad+\lambda(1-\lambda)\left(\sum_{2 \leq i<j \leq p} d\left(x_{i}, y_{j}\right)+d\left(y_{i}, x_{j}\right)\right)+(1-\lambda)^{2} \sum_{2 \leq i<j \leq p} d\left(y_{i}, y_{j}\right) \\
& \leq \lambda \sum_{1 \leq i j \leq p} d\left(x_{i}, x_{j}\right)+(1-\lambda)\left(\sum_{i=2}^{p} d\left(\left(y_{i}, x_{1}\right)+\sum_{2 \leq i<j \leq p} d\left(y_{i}, y_{j}\right)\right)\right. \\
& =\lambda D_{p}\left(x_{1}, x_{2}, \ldots, x_{p}\right)+(1-\lambda) D_{p}\left(x_{1}, y_{2}, \ldots, y_{p}\right) \leq \Lambda .
\end{aligned}
$$

That means

$$
\begin{aligned}
& \left(W\left(x_{2}, y_{2}, \lambda\right), W\left(x_{3}, y_{3}, \lambda\right), \ldots W\left(x_{p}, y_{p}, \lambda\right)\right) \\
& \quad=W\left(\Pi_{i=2}^{p} C_{i}\right)\left(\left(x_{2}, x_{3}, \ldots, x_{p}\right),\left(y_{2}, y_{3}, \ldots y_{p}\right), \lambda\right) \in B_{1},
\end{aligned}
$$

for all $\left(x_{2}, x_{3}, \ldots, x_{p}\right),\left(y_{2}, y_{3}, \ldots, y_{p}\right) \in B_{1}$ and $\lambda \in I$.

Define

$$
\widetilde{T}: \cup_{i=1}^{p}\left(\prod_{j=i}^{p+i-2} A_{j}\right) \longrightarrow \cup_{i=1}^{p}\left(\prod_{j=i}^{p+i-2} A_{j}\right),
$$

by

$$
\widetilde{T}\left(x_{1}, x_{2}, \ldots, x_{p-1}\right)=\left(T_{x_{1}}, T_{x_{2}}, \ldots, T_{x_{p-1}}\right) .
$$

We have $\widetilde{T}$ which is pcyclic:

$$
\begin{aligned}
& \widetilde{T}\left(\prod_{i=1}^{p-1} A_{i}\right) \subseteq\left(\prod_{i=2}^{p} A_{i}\right), \widetilde{T}\left(\prod_{i=2}^{p} A_{i}\right) \\
& \quad \subseteq\left(\prod_{i=3}^{p} A_{i} \times A_{1}\right), \ldots, \widetilde{T}\left(A_{P} \times \prod_{i=1}^{p-2} A_{i}\right) \subseteq\left(\prod_{i=1}^{p-1} A_{i}\right) .
\end{aligned}
$$

$$
=\left(T_{x_{1}}, T_{x_{2}} \ldots, T_{x_{p-1}}\right) \in\left(\Pi_{i=2}^{p} C_{i}\right) \text {. }
$$

We have

$$
\Delta\left(T_{x_{1}}, T_{x_{2}}, \ldots, T_{x_{p-1}}\right)\left(C_{1}\right)
$$

$$
\leq \Lambda \Rightarrow \widetilde{T}\left(x_{1}, x_{2}, \ldots, x_{p-1}\right) \in B_{1} \Rightarrow \widetilde{T}\left(\prod_{i=1}^{p-1} C_{i}\right) \subseteq B_{1}
$$

So $\widetilde{T}$ is $p$ cyclic on $\cup_{i=1}^{P} B_{i}$. $\left(\left(\Pi_{i=1}^{p-1} C_{i}\right),\left(\Pi_{i=2}^{p} C_{i}\right), \ldots,\left(C_{P} \times\right.\right.$ $\left.\left.\Pi_{i=1}^{p-2} C_{i}\right)\right)$ is maximal in $\tilde{\sum}$ where

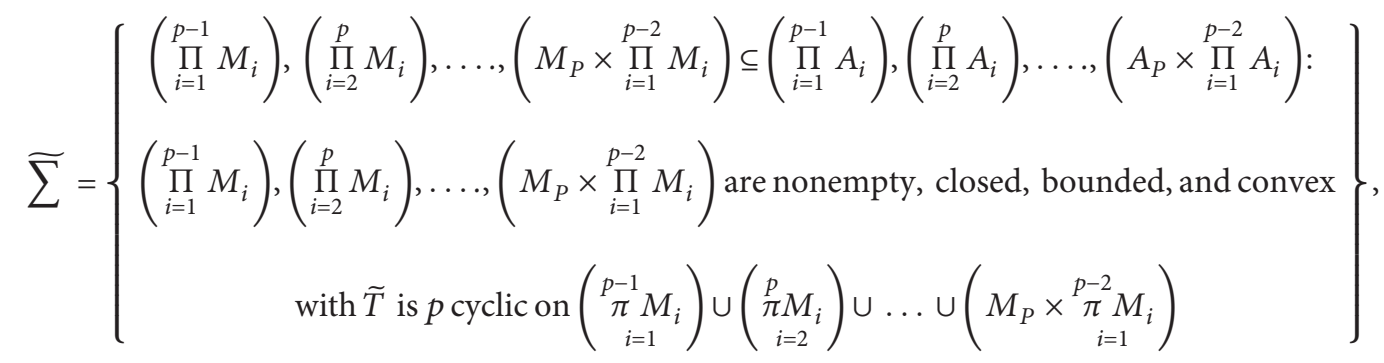


$\tilde{\Sigma}$ is partially ordered by $M_{1 i} \tilde{\leq} M_{2 i} \Leftrightarrow M_{2 i} \subseteq M_{1 i}$. For $i=1, \ldots, p$. Let $\left(M_{i j}\right)_{j \in I i=1, \ldots ., p}$ be an increasing chain of $\tilde{\Sigma}$, and since $X$ has a the $(C)$ property, $\cap_{j \in I} M_{i j}$ for ${ }_{i=1, \ldots, p}$ are bounded, closed, and convex subsets. We have

$$
M_{i j} \tilde{\leq} \bigcap_{j \in I} M_{i j} \text { for }_{i=1, \ldots, p} \text { forall } j \in I .
$$

So

$$
\begin{aligned}
B_{1} & =\left(C_{2}, \ldots, C_{p}\right), \\
B_{i} & =\left(C_{i+1}, \ldots, C_{p+i-1}\right) \text { for } i=2, \ldots, p .
\end{aligned}
$$

Inductively,

$$
\Delta_{\left(x_{1}, x_{2}, \ldots x_{p-1}\right)}\left(C_{p}\right) \leq \Lambda, \forall\left(x_{1}, x_{2}, \ldots, x_{p-1}\right) \in\left(\prod_{i=1}^{p-1} C_{i}\right) \text {. }
$$

So, we have

$$
\begin{gathered}
\Delta_{\left(x_{1}, x_{2}, \ldots, x_{p-1}\right)}\left(C_{p}\right)-k \Delta\left(\left(\prod_{i=1}^{p} C_{i}\right)\right) \leq(1-k) \sigma\left(\left(\prod_{i=1}^{p} A_{i}\right)\right), \\
\forall\left(x_{1}, x_{2}, \ldots, x_{p-1}\right) \in\left(\prod_{i=1}^{p-1} C_{i}\right) \\
\Rightarrow(1-k) \Delta\left(\left(\prod_{i=1}^{p} C_{i}\right)\right) \leq(1-k) \sigma\left(\prod_{i=1}^{p} A_{i}\right) .
\end{gathered}
$$

So

$$
\Delta\left(\prod_{i=1}^{p} C_{i}\right) \leq \sigma\left(\prod_{i=1}^{p} A_{i}\right) .
$$

Now let $\left(r_{1}, r_{2}, \ldots, r_{p}\right) \in\left(\Pi_{i=1}^{p} C_{i}\right)$, then we have

$$
\begin{aligned}
\sigma\left(\prod_{i=1}^{p} A_{i}\right) & \leq D_{p}\left(r_{1}, T r_{1}, T^{2} r_{1}, \ldots, T^{p-1} r_{1}\right), D_{p}\left(T^{p-1} r_{2}, r_{2}, T r_{2}, T^{2} r_{2}, \ldots, T^{p-2} r_{2}\right), \ldots, D_{p}\left(T r_{p}, T^{2} r_{p}, \ldots, T^{p-1} r_{p}, r_{p}\right) \\
& \leq \Delta\left(\prod_{i=1}^{p} C_{i}\right) \leq \sigma\left(\prod_{i=1}^{p} A_{i}\right), \text { then } \Delta\left(\prod_{i=1}^{p} C_{i}\right)=\sigma\left(\prod_{i=1}^{p} A_{i}\right),
\end{aligned}
$$

which finishes the proof of the theorem.

Now, we give some examples for $p=4$.

Example 1. Let $X$ be $\mathbb{R}^{2}$ normed by the norm $\|(x, y)\|=$ $|x|+|y|$, and let $A=[1,2] \times\{0\}, \quad B=\{0\} \times[-2,-1]$, $C=[-2,-1] \times\{0\}, \quad$ and $\quad D=\{0\} \times[1,2], \quad$ then
$\sigma(A, B, C, D)=D_{4}((1,0),(0,-1),(-1,0),(0,1))=12$. We define $W: X \times X \times I \longrightarrow X$ by $W(x, y, \lambda)=\lambda x+(1-\lambda) y$ for all $x, y \in X, \lambda \in I$, then $(X, d, W)$ is a complete convex metric space. $W$ is a $(S)$ convex structure on $\mathrm{X}$. Let $a, b \in X, x=W(a, b, \alpha)$, and $y=W(a, b, \beta)$ with $\alpha>\beta$. We have 


$$
\begin{aligned}
W\left(a, y, \frac{\alpha-\beta}{1-\beta}\right) & =\frac{\alpha-\beta}{1-\beta} a+\left(1-\frac{\alpha-\beta}{1-\beta}\right) y=\frac{\alpha-\beta}{1-\beta} a+\left(\frac{1-\alpha}{1-\beta}\right) y \\
& =\frac{\alpha-\beta}{1-\beta} a+\left(\frac{1-\alpha}{1-\beta}\right) W(a, b, \beta) \\
& =\frac{\alpha-\beta}{1-\beta} a+\left(\frac{1-\alpha}{1-\beta}\right)(\beta a+(1-\beta) b)=\frac{1}{1-\beta}(\alpha(1-\beta)) a+(1-\alpha) b=\alpha a+(1-\alpha) b=x, \\
W\left(x, b, \frac{\beta}{\alpha}\right) & =\frac{\beta}{\alpha} x+\left(1-\frac{\beta}{\alpha}\right) b=\frac{\beta}{\alpha} W(a, b, \alpha)+\left(1-\frac{\beta}{\alpha}\right) b \\
& =\frac{\beta}{\alpha}(\alpha a+(1-\alpha) b)+\left(1-\frac{\beta}{\alpha}\right) b=\beta a+\frac{\beta}{\alpha}((1-\alpha) b)+\left(1-\frac{\beta}{\alpha}\right) b \\
& =\beta a+(1-\beta) b=y .
\end{aligned}
$$

Put

$T: A \cup B \cup C \cup D \longrightarrow A \cup B \cup C \cup D$,

such that

$$
\begin{aligned}
& T(x, 0)=\left(0,-\frac{x+1}{2}\right) \quad \text { if } x \in[1,2], \\
& T(0, y)=\left(\frac{y-1}{2}, 0\right) \quad \text { if } y \in[-2,-1],
\end{aligned}
$$

$$
\begin{aligned}
& T(z, 0)=\left(0, \frac{1-z}{2}\right) \quad \text { if } z \in[-2,-1], \\
& T(0, t)=\left(\frac{t+1}{2}, 0\right) \quad \text { if } t \in[1,2] .
\end{aligned}
$$

We have

$$
T(A) \subseteq B, T(B) \subseteq C, T(C) \subseteq D, T(D) \subseteq A,
$$

$$
\begin{aligned}
D_{4}(T(x, 0), T(0, y), T(z, 0), T(0, t))= & \left(\frac{x+1}{2}+\frac{1-y}{2}\right)+\left(\frac{x+1}{2}+\frac{1-z}{2}\right) \\
& +\left(\frac{x+1}{2}+\frac{t+1}{2}\right)+\left(\frac{1-z}{2}+\frac{1-y}{2}\right)+\left(\frac{t+1}{2}+\frac{1-y}{2}\right)+\left(\frac{t+1}{2}+\frac{1-z}{2}\right) \\
= & \frac{1}{2}(x-y)+(x-z)+(x+t)+(-y-z)+(t-y)+(t-z)+6 \\
= & \frac{1}{2} D_{4}((x, 0),(0, y),(z, 0),(0, t))+6 \\
= & \frac{1}{2} D_{4}((x, 0),(0, y),(z, 0),(0, t))+\left(1-\frac{1}{2}\right) \sigma(A, B, C, D) .
\end{aligned}
$$

So $T$ is a quadricyclic contraction. Then, $T$ has $(1,0)$ a best proximity point, since

$$
\begin{aligned}
& D_{4}\left((1,0), T(1,0), T^{2}(1,0), T^{3}(1,0)\right) \\
& \quad=D_{4}((1,0),(0,-1),(-1,0),(0,1))=12=\sigma(A, B, C, D) .
\end{aligned}
$$

Example 2. Let $k \in(0,1)$, and let $A, B, C$, and $D$ the four subsets of $l_{p}, 1 \leq p \leq \infty$, defined by

$$
\begin{aligned}
& A=\left\{\left(1+k^{4 n}\right) e_{4 n}, \quad n \in \mathbb{N}\right\}, \\
& B=\left\{\left(1+k^{4 m-3}\right) e_{4 m-3}, \quad m \in \mathbb{N}\right\}, \\
& C=\left\{\left(1+k^{4 s-2}\right) e_{4 s-2}, \quad s \in \mathbb{N}\right\}, \\
& D=\left\{\left(1+k^{4 t-1}\right) e_{4 t-1}, \quad t \in \mathbb{N}\right\} .
\end{aligned}
$$

Put

$$
T: A \cup B \cup C \cup D \longrightarrow A \cup B \cup C \cup D,
$$

such that $T\left(1+k^{p}\right) e_{p}=\left(1+k^{p+1}\right) e_{p+1}$ for all $p \in \mathbb{N}$.

We have $T(A) \subset B, T(B) \subset C, T(C) \subset D$, and $T(D) \subset A$. 
Since $k \in(0,1), \lim \left(\left(1+k^{4 n-i}\right)^{p}+\left(1+k^{4 n-j}\right)^{p}\right)^{1 / p}=\quad$ So $\sigma(A, B, C, D)=6.2^{1 / p}$. $2^{1 / p}$ for $i, j=1,2,3$.

$$
\begin{aligned}
& D_{4}\left(T\left(1+k^{4 n}\right), T\left(1+k^{4 m-3}\right), T\left(1+k^{4 s-2}\right), T\left(1+k^{4 t-1}\right)\right) \\
& =D_{4}\left(\left(1+k^{4 n+1}\right),\left(1+k^{4 m-2}\right),\left(1+k^{4 s-1}\right),\left(1+k^{4 t}\right)\right) \\
& =\left(\left(1+k^{4 n+1}\right)^{p}+\left(1+k^{4 m-2}\right)^{p}\right)^{1 / p}+\left(\left(1+k^{4 n+1}\right)^{p}+\left(1+k^{4 s-1}\right)^{p}\right)^{1 / p} \\
& +\left(\left(1+k^{4 n+1}\right)^{p}+\left(1+k^{4 t}\right)^{p}\right)^{1 / p}+\left(\left(1+k^{4 m-2}\right)^{p}+\left(1+k^{4 s-1}\right)^{p}\right)^{1 / p} \\
& +\left(\left(1+k^{4 m-2}\right)^{p}+\left(1+k^{4 t}\right)^{p}\right)^{1 / p}+\left(\left(1+k^{4 s-1}\right)^{p}+\left(1+k^{4 t}\right)^{p}\right)^{1 / p} \\
& =\left(\left(k\left(1+k^{4 n}\right)+(1-k)\right)^{p}+\left(k\left(1+k^{4 m-3}\right)+(1-k)\right)^{p}\right)^{1 / p} \\
& +\left(\left(k\left(1+k^{4 n}\right)+(1-k)\right)^{p}+\left(k\left(1+k^{4 s-2}\right)+(1-k)\right)^{p}\right)^{1 / p} \\
& +\left(\left(k\left(1+k^{4 n}\right)+(1-k)\right)^{p}+\left(k\left(1+k^{4 t-1}\right)+(1-k)\right)^{p}\right)^{1 / p} \\
& +\left(\left(k\left(1+k^{4 m-3}\right)+(1-k)\right)^{p}+\left(k\left(1+k^{4 s-2}\right)+(1-k)\right)^{p}\right)^{1 / p} \\
& +\left(\left(k\left(1+k^{4 m-3}\right)+(1-k)\right)^{p}+\left(k\left(1+k^{4 t-1}\right)+(1-k)\right)^{p}\right)^{1 / p} \\
& +\left(\left(k\left(1+k^{4 s-2}\right)+(1-k)\right)^{p}+\left(k\left(1+k^{4 t-1}\right)+(1-k)\right)^{p}\right)^{1 / p} \\
& \leq\left(\left(k\left(1+k^{4 n}\right)\right)^{p}+\left(k\left(1+k^{4 m-3}\right)\right)^{p}\right)^{1 / p}+\left(2(1-k)^{p}\right)^{1 / p} \\
& +\left(\left(k\left(1+k^{4 n}\right)\right)^{p}+\left(k\left(1+k^{4 s-2}\right)\right)^{p}\right)^{1 / p}+\left(2(1-k)^{p}\right)^{1 / p} \\
& \left(\left(k\left(1+k^{4 n}\right)\right)^{p}+\left(k\left(1+k^{4 t-1}\right)\right)^{p}\right)^{1 / p}+\left(2(1-k)^{p}\right)^{1 / p} \\
& +\left(\left(k\left(1+k^{4 m-3}\right)\right)^{p}+\left(k\left(1+k^{4 s-2}\right)\right)^{p}\right)^{1 / p}+\left(2(1-k)^{p}\right)^{1 / p} \\
& +\left(\left(k\left(1+k^{4 m-3}\right)\right)^{p}+\left(k\left(1+k^{4 t-1}\right)\right)^{p}\right)^{1 / p}+\left(2(1-k)^{p}\right)^{1 / p} \\
& +\left(\left(k\left(1+k^{4 s-2}\right)\right)^{p}+\left(k\left(1+k^{4 t-1}\right)\right)^{p}\right)^{1 / p}+\left(2(1-k)^{p}\right)^{1 / p} \\
& \leq\left(\left(k\left(1+k^{4 n}\right)\right)^{p}+\left(k\left(1+k^{4 m-3}\right)\right)^{p}\right)^{1 / p} \\
& +\left(\left(k\left(1+k^{4 n}\right)\right)^{p}+\left(k\left(1+k^{4 s-2}\right)\right)^{p}\right)^{1 / p} \\
& \left(\left(k\left(1+k^{4 n}\right)\right)^{p}+\left(k\left(1+k^{4 t-1}\right)\right)^{p}\right)^{1 / p} \\
& +\left(\left(k\left(1+k^{4 m-3}\right)\right)^{p}+\left(k\left(1+k^{4 n s-2}\right)\right)^{p}\right)^{1 / p} \\
& +\left(\left(k\left(1+k^{4 m-3}\right)\right)^{p}+\left(k\left(1+k^{4 t-1}\right)\right)^{p}\right)^{1 / p} \\
& +\left(\left(k\left(1+k^{4 s-2}\right)\right)^{p}+\left(k\left(1+k^{4 t-1}\right)\right)^{p}\right)^{1 / p}+6.2^{1 / p}(1-k) \\
& =k D_{4}\left(\left(1+k^{4 n}\right),\left(1+k^{4 m-3}\right),\left(1+k^{4 s-2}\right),\left(1+k^{4 t-1}\right)\right)+(1-k) \sigma(A, B, C, D) \text {. }
\end{aligned}
$$


$\mathrm{T}$ is a quadricyclic contraction.

Let

Put $W: \quad l_{p} \times l_{p} \times I \longrightarrow l_{p}$ such that $W(x, y, \lambda)=$ $((\lambda x+(1-\lambda) y) /\|\lambda x+(1-\lambda) y\|)$.

$$
\begin{aligned}
u \in l_{p}, x, y \in l_{p}, d(u, W(x, y, \lambda)) & =d(u,((\lambda x+(1-\lambda) y) /(\|\lambda x+(1-\lambda) y\|))) \\
& =\frac{1}{\|\lambda x+(1-\lambda) y\|} d(u, \lambda x+(1-\lambda) y) \\
& =\frac{1}{\|\lambda x+(1-\lambda) y\|}\left(\sum\left|u_{i}+\lambda x_{i}+(1-\lambda) y_{i}\right|^{p}\right)^{1 / p} \\
& =\frac{1}{\|\lambda x+(1-\lambda) y\|}\left(\sum\left|\lambda u_{i}+(1-\lambda) u_{i}+\lambda x_{i}+(1-\lambda) y_{i}\right|^{p}\right)^{1 / p} \\
& =\frac{1}{\|\lambda x+(1-\lambda) y\|}\left(\sum\left|\lambda\left(u_{i}+x_{i}\right)+(1-\lambda)\left(u_{i}+y_{i}\right)\right|^{p}\right)^{1 / p} \\
& \leq \frac{1}{\|\lambda x+(1-\lambda) y\|}\left(\lambda^{p} \sum \mid\left(u_{i}+\left.x_{i}\right|^{p}+(1-\lambda)^{p} \sum\left|u_{i}+y_{i}\right|^{p}\right)^{1 / p}\right. \\
& \leq \frac{1}{\|\lambda x+(1-\lambda) y\|} \lambda\left(\sum\left|\left(u_{i}+\left.x_{i}\right|^{p}\right)^{1 / p}+(1-\lambda) \sum\right| u_{i}+\left.y_{i}\right|^{p}\right)^{1 / p} \\
& \leq \lambda d(u, x)+(1-\lambda) d(u, y) .
\end{aligned}
$$

Then, $\left(l_{p}, d, W\right)$ is a complete convex metric space for all $1 \leq p \leq \infty$.

For $\quad p=2, \quad$ let $\quad a=(1,0,0,0, \ldots) \in l_{p}, \quad b=$ $(0,1,0,0, \ldots) \in l_{p}$,

$$
\begin{aligned}
& x=W\left(a, b, \frac{1}{2}\right)=\left(\frac{1}{\sqrt{2}}, \frac{1}{\sqrt{2}}, 0,0,0, \ldots\right), \\
& y=W\left(a, b, \frac{1}{4}\right)=\left(\frac{1}{\sqrt{10}}, \frac{3}{\sqrt{10}}, 0,0,0, \ldots\right) .
\end{aligned}
$$

We have

$W(a, y,(1 / 3))=((1 / 3)+(2 /(3 \sqrt{10})),(2 / \sqrt{10}), 0,0, \ldots) \neq x$,

$W(x, b,(1 / 2))=((1 /(2 \sqrt{2})),(1 /(2 \sqrt{2}))+(1 / 2), 0,0,0, \ldots) \neq y$.

$W$ is not a $(S)$ convex structure on $X$. Then, $T$ has not a best proximity point.

\section{Data Availability}

No data were used to support this study.

\section{Conflicts of Interest}

The authors declare that there are no conflicts of interest regarding the publication of this paper.

\section{References}

[1] W. A. Kirk, P. S. Srinivasan, and P. Veeramani, "Fixed point fo mappings satisfyaing cyclical contractive conditions," Fixed Point Theory, vol. 4, pp. 79-89, 2003.

[2] A. A. Eldred and P. Veeramani, "Existence and convergence of best proximity points," Journal of Mathematical Analysis and Applications, vol. 323, no. 2, pp. 1001-1006, 2006.

[3] M. Gabeleh and N. Shahzad, "Some new results on cyclic relatively nonexpansive mapping in convex metric," Journal of Inequalities and Application, vol. 2014, p. 350, 2014.

[4] L. A. Talman, "Fixed points for condensing multifunctions in metric spaces with convex structure," Kodai Mathematical Seminar Reports, vol. 29, no. 1-2, pp. 62-70, 1977.

[5] M. A. Al-Thagafi and N. Shahzad, "Convergence and existence results for best proximity points," Nonlinear Analysis: Theory, Methods \& Applications, vol. 70, no. 10, pp. 3665-3671, 2009.

[6] S. Sadiq Basha, "Best proximity points theorems," J. Approx. Theory, vol. 163, pp. 1772-1781, 2011.

[7] A. Felhi and H. Aydi, "Best Proximity Points and stability results for controlled proximal contractive set valued mapping," Fixed Point Theory and Applications, vol. 2016, p. 22, 2016.

[8] S. Karpagam and S. Agrawal, "Best proximity points theorems for cyclic Meir-Keeler contraction maps," Nonlinear Analysis, vol. 74, no. 4, pp. 1040-1046, 2011.

[9] T. Sabar, M. Aamri, and A. Bassou, "Best proximity point of tricyclic contraction," Advances in Fixed Point Theory, vol. 7, pp. 512-523, 2017. 
[10] T. Sabar, A. Bassou, and M. Aamri, "Best proximity points for tricyclic contractions in a (S) convex metric spaces," International Journal of Mathematical Analysis, vol. 12, no. 6, pp. 289-299, 2018.

[11] A. A. Abdelhakim, "A convexity of functions on convex metric spaces of Takahashi and applications," Journal of the Egyptian Mathematical Society, vol. 24, no. 3, pp. 348-354, 2016.

[12] W. Takahashi, "A convexity in metric space and nonexpansive mappings I," Kodai Mathematical Seminar Reports, vol. 22, no. 2, pp. 142-149, 1970. 